\title{
Displasia broncopulmonar
}

\author{
Bronchopulmonary dysplasia \\ Luciana F. Velloso Monte ${ }^{1}$, Luiz Vicente F. da Silva Filho², \\ Milton Harumi Miyoshi ${ }^{3}$, Tatiana Rozov 4
}

\section{Resumo}

Objetivo: Apresentar uma ampla revisão da literatura sobre displasia broncopulmonar, abordando novas definições, fisiopatologia, prevenção, tratamento, prognóstico e evolução.

Fonte dos dados: Foram selecionados os artigos mais relevantes sobre o tema, desde a sua descrição inicial, em 1967, pesquisados na MEDLINE.

Síntese dos dados: A displasia broncopulmonar é considerada uma das principais causas de doença pulmonar crônica em lactentes. Está associada a hospitalizações freqüentes e prolongadas, especialmente por doenças pulmonares, altos índices de mortalidade e alterações no desenvolvimento neuropsicomotor e no crescimento pônderoestatural. A patogênese é complexa e influenciada principalmente por prematuridade, infecção, oxigênio suplementar e ventilação mecânica. A prevenção envolve o acompanhamento pré-natal adequado, a prevenção do parto prematuro, o uso pré-natal do corticosteróide, a terapia de reposição de surfactante e o uso de estratégias ventilatórias "protetoras". O tratamento do paciente com displasia broncopulmonar demanda uma equipe multidisciplinar. Quando indicada, a suplementação de oxigênio é de extrema importância. Apesar de maior risco de morbimortalidade nos primeiros anos de vida, a evolução em longo prazo é favorável na maioria das vezes.

Conclusões: A displasia broncopulmonar vem sendo profundamente estudada na tentativa de identificação das suas causas e possibilidades de prevenção e de tratamento. Ainda existem controvérsias quanto a esses assuntos e também em relação ao prognóstico desses pacientes, especialmente quando se trata da evolução tardia da "nova" displasia broncopulmonar.

J Pediatr (Rio J). 2005;81(2):99-110: Displasia broncopulmonar, oxigenoterapia, ventilação mecânica, corticosteróide, prevenção, tratamento, evolução.

\begin{abstract}
Objective: To present a wide-ranging review of the literature on bronchopulmonary dysplasia, covering new definitions, pathophysiology, prevention, treatment, prognosis and progression.
\end{abstract}

Sources of data: The most relevant articles published on the subject since it was first described in 1967 were selected from MEDLINE search results.

Summary of the findings: Bronchopulmonary dysplasia is considered one of the primary causes of chronic lung disease among infants. It is associated with frequent and prolonged hospital admissions, in particular for pulmonary diseases, with high rates of mortality and alterations to neuropsychomotor development and pondero-statural growth. Pathogenesis is complex, being primarily influenced by prematurity, infection, supplementary oxygen and mechanical ventilation. Prevention involves appropriate prenatal care, the prevention of premature delivery, prenatal corticosteroids, surfactant replacement therapy and "protective" ventilatory strategies. Treatment of bronchopulmonary dysplasia patients demands a multidisciplinary team. When indicated, oxygen supplementation is extremely important. Despite increased risk of morbidity and mortality during the first years of life, long term progress is favorable in the majority of cases.

Conclusions: Bronchopulmonary dysplasia has been and continues to be studied in great depth with the objective of identifying its causes and possible prevention and treatment strategies. Controversies remain with respect of these issues and also about the prognosis of these patients, in particular when the subject is long-term progress of "new" bronchopulmonary dysplasia patients.

J Pediatr (Rio J). 2005;81(2):99-110: Bronchopulmonary dysplasia, oxygen therapy, mechanical ventilation, corticosteroid, prevention, treatment, progression.

1. Mestranda, Departamento de Pediatria, Universidade de São Paulo. Médica assistente, Unidade de Pneumologia Pediátrica e Pronto Atendimento do Instituto da Criança Professor Pedro de Alcântara, Hospital das Clínicas, Faculdade de Medicina, Universidade de São Paulo (USP), São Paulo, SP.

2. Mestre e Doutor em Pediatria. Médico assistente, Unidade de Pneumologia, Instituto da Criança Professor Pedro de Alcântara, Hospital das Clínicas, Faculdade de Medicina,USP, São Paulo, SP.

3. Professor assistente, Departamento de Pediatria, Universidade Federal de São Paulo (UNIFESP/EPM). Consultor médico, Unidade de Terapia Intensiva Neonatal, Hospital e Maternidade Santa Joana, São Paulo, SP.

4. Professora de Pós-graduação, Departamento de Pediatria e de Reabilitação Pulmonar, UNIFESP/EPM. Livre-docente, Departamento de Pediatria, Faculdade de Medicina, USP, São Paulo, SP.

Artigo submetido em 06.02.04, aceito em 14.04.04.

Como citar este artigo: Monte LF, Silva Filho LV, Miyoshi MH, Rozov T. Displasia broncopulmonar. J Pediatr (Rio J). 2005;81:99-110. 


\section{Introdução}

A displasia broncopulmonar (DBP) é uma doença pulmonar crônica com características clínicas, radiológicas e histológicas próprias. Acomete, em geral, os recém-nascidos prematuros submetidos a oxigenoterapia e ventilação mecânica nos primeiros dias de vida. Desde a sua descrição, na década de 60 , observou-se um grande progresso na assistência perinatal, como o maior uso de corticóide antenatal, a viabilização da terapêutica com o surfactante exógeno e o surgimento de novas técnicas de monitoração não-invasiva e de ventilação mecânica. Tais fatos contribuíram para o aumento da sobrevida de recém-nascidos cada vez mais imaturos e, em conseqüência, da incidência da DBP. Estima-se que cerca de 3.000 a 7.000 neonatos sejam afetados anualmente pela doença nos EUA. Apesar da freqüência da doença não ter diminuído nas últimas décadas, o avanço no tratamento de recém-nascidos com insuficiência respiratória tem atenuado a sua gravidade. Apesar disso, a DBP ainda tem grande importância clínica e de saúde pública, já que atualmente é reconhecida como uma das principais causas de doença respiratória crônica na infância, levando a hospitalizações freqüentes e prolongadas com altos índices de mortalidade e alterações do crescimento pôndero-estatural e desenvolvimento neuropsicomotor ${ }^{1-3}$. Tamanho impacto justifica o grande investimento nas pesquisas para identificar suas causas e buscar alternativas para prevenção e tratamento. Este artigo revisa os aspectos mais importantes envolvidos na fisiopatologia da DBP, além de tópicos importantes para a sua prevenção, tratamento e acompanhamento clínico.

\section{Definição}

A DBP foi descrita inicialmente em 1967 por Northway et al. ${ }^{4}$ como uma doença pulmonar crônica que acometia recém-nascidos prematuros com síndrome do desconforto respiratório (SDR), ou doença da membrana hialina, submetidos à ventilação mecânica prolongada com níveis pressóricos e frações inspiradas de oxigênio $\left(\mathrm{FiO}_{2}\right)$ elevados. $\mathrm{Na}$ época, a doença foi classificada em quatro estágios de acordo com as características clínicas, radiológicas e anatomopatológicas. Os pacientes cursavam com taquidispnéia e hipoxemia em ar ambiente, lesões císticas e hipertransparentes com traves radiopacas à radiografia de tórax, cardiomegalia e cor pulmonale, além de metaplasia e fibrose pulmonar. Boa parte deles era dependente de oxigênio por meses ou anos. Em 1969, Pursey et al. ${ }^{5}$ descreveram alterações pulmonares difusas relacionadas com a ventilação mecânica prolongada em recém-nascidos com doença pulmonar de diversas etiologias, incluindo aqueles que não tinham SDR, sugerindo que esta não necessariamente seria a precursora da DBP.

Em 1979, Bancalari et al. ${ }^{6}$ definiram a DBP como insuficiência respiratória no neonato que necessitou de pelo menos 3 dias de ventilação mecânica e que evoluía dependente de oxigênio por mais de 28 dias de vida, apresentando sinais de aumento de trabalho respiratório e alterações radiológicas pulmonares. Esse critério foi o mais utilizado na década de 80 e início dos anos 90.
Entretanto, os avanços nos cuidados perinatais nas últimas décadas proporcionaram maior sobrevida de recém-nascidos cada vez mais imaturos, em especial os com peso de nascimento abaixo de 1.000 gramas. Esses pacientes habitualmente são dependentes de oxigênio e/ou ventilação mecânica aos 28 dias de vida sem, no entanto, apresentarem comprometimento pulmonar importante. Além disso, muitos desses se tornam assintomáticos e livres da suplementação do oxigênio antes da alta hospitalar. Com essa preocupação, Shennan et al. ${ }^{7}$, em 1988, sugeriram uma nova definição com o objetivo de identificar os neonatos com maior chance de desenvolverem problemas pulmonares crônicos. Os autores introduziram o termo "doença pulmonar crônica do prematuro", definindo-a como persistência de sinais e sintomas respiratórios acompanhados de alterações radiológicas pulmonares necessitando de oxigênio suplementar além de 36 semanas de idade pós-conceptual, o que poderia ter uma maior correlação com o prognóstico em longo prazo.

No entanto, nenhuma dessas definições determina com precisão a gravidade da lesão pulmonar. Com as mudanças no perfil epidemiológico e nas características clínicas e histopatológicas da doença, realizou-se recentemente uma conferência de consenso nos EUA organizada pelo Instituto Nacional de Saúde da Criança e Desenvolvimento Humano (NICHD) e Instituto Nacional do Sangue, Pulmão e Coração (NHLBI) em conjunto com o Departamento de Doenças Raras (ORD), com objetivo de estabelecer uma uniformização na terminologia, definir critérios de gravidade e instituir estratégias de prevenção e tratamento para a DBP8. A fim de evitar confusão acerca da terminologia, a conferência de consenso aprovou a fixação do termo "displasia broncopulmonar" em detrimento de "doença pulmonar crônica", por causa da distinção clara dessa doença, em termos epidemiológicos, etiopatogênicos e prognósticos, com as outras múltiplas formas de pneumopatias crônicas que ocorrem na infância. Além disso, foram propostos novos critérios diagnósticos e de avaliação da gravidade da DBP. Assim, a DBP deve ser considerada em qualquer neonato que permanece dependente de oxigênio em concentrações acima de $21 \%$ por um período maior ou igual a 28 dias. Tal paciente, de acordo com a idade gestacional de nascimento, deve ser submetido à reavaliação diagnóstica e à determinação da gravidade da doença, de acordo com a Tabela 1. As alterações radiológicas, apesar de comumente presentes, foram consideradas de interpretação inconsistente, não sendo utilizadas para a definição ou avaliação da gravidade da DBP, de acordo com o consenso.

\section{Incidência}

A incidência da DBP é inversamente proporcional à idade gestacional e ao peso de nascimento. Sua ocorrência é pouco comum em neonatos com idade gestacional superior a 34 semanas, apesar de existirem casos descritos em recém-nascidos a termo. Com os avanços na assistência perinatal, a incidência da forma grave ou "clássica" da DBP diminuiu consideravelmente. Além disso, os relatos na literatura são extremamente variáveis de centro para cen- 
Tabela 1 - Critérios diagnósticos e de classificação da gravidade da DBP8

\begin{tabular}{|c|c|c|}
\hline \multirow{2}{*}{$\begin{array}{l}\text { RN dependente de } \mathrm{O}_{2} \text { suplementar } \\
\text { aos } 28 \text { dias de vida }\end{array}$} & \multicolumn{2}{|c|}{ Idade gestacional ao nascimento } \\
\hline & $<32$ semanas & $\geq \mathbf{3 2}$ semanas \\
\hline Época de reavaliação * & 36 semanas de IPM ou à $\mathrm{AH}^{+}$ & 56 dias de vida ou à $\mathrm{AH}^{+}$ \\
\hline DBP leve & Ar ambiente & Ar ambiente \\
\hline DBP moderada & $\mathrm{Em} \mathrm{FiO}_{2}<0,30 \neq \S$ & $\mathrm{Em} \mathrm{FiO}_{2}<0,30 \neq \S$ \\
\hline DBP grave & $\begin{array}{c}\mathrm{Em} \mathrm{FiO}_{2} \geq 0,30 \text { e/ou } \\
\mathrm{CPAP} \text { ou } \mathrm{VM} \neq \S\end{array}$ & $\begin{array}{c}\mathrm{Em} \mathrm{FiO}_{2} \geq 0,30 \text { e/ou } \\
\mathrm{CPAP} \text { ou } \mathrm{VM} \neq \S\end{array}$ \\
\hline \multicolumn{3}{|c|}{$\begin{array}{l}\mathrm{RN}=\text { recém-nascido; } \mathrm{IPM}=\text { idade pós-mentrual; } \mathrm{AH}=\text { alta hospitalar; } \mathrm{DBP}=\text { displasia broncopulmonar; } \mathrm{FiO}_{2}=\text { fração de oxigênio } \\
\text { inspirada; } \mathrm{CPAP}=\text { pressão positiva contínua na via aérea: } \mathrm{VM}=\text { ventilacão mecânica. }\end{array}$} \\
\hline \multicolumn{3}{|c|}{$\begin{array}{l}\text { * Na data da reavaliação, a necessidade de oxigênio e/ou suporte ventilatório (CPAP nasal ou VM) suplementar não deve refleti } \\
\text { um evento agudo, mas um estado basal em que o paciente esteja recebendo a terapia por vários dias. Além disso, recomenda- } \\
\text { se a realização de um teste fisiológico para confirmar a real necessidade da oxigenoterapia e/ou do suporte ventilatório } \\
\text { suplementar. Não há, ainda, um consenso sobre a melhor técnica para realizar tal teste. } \\
\text { † Considerar o que ocorrer primeiro. } \\
\text { f Não há relatos do modo pelo qual se faz a mensuração da } \mathrm{FiO}_{2} \text {. } \\
\text { § Não considerar os pacientes que recebem oxigênio e/ou suporte ventilatório suplementar para o tratamento de distúrbios não- } \\
\text { pulmonares (p.ex.: apnéia central, paralisia diafragmática etc.), a menos que desenvolvam alterações no parênquima pulmonar } \\
\text { e desconforto respiratório. }\end{array}$} \\
\hline
\end{tabular}

tro, devido às diferenças da população estudada, à falta de padronização das estratégias ventilatórias empregadas no tratamento da doença pulmonar aguda, à grande variabilidade no manuseio da oferta hídrica e na prática de cuidados de RN criticamente doentes e aos diferentes critérios diagnósticos utilizados para definir a doença (Tabela 2) 9-12. Em nosso meio, Cunha et al. ${ }^{13}$ encontraram uma incidência de DBP de $26,6 \%$ na avaliação de 124 crianças com peso de nascimento abaixo de $1.500 \mathrm{~g}$ que sobreviveram aos 28 dias de vida entre os anos de 2000 e 2002.

Tabela 2 - Percentual de pacientes com displasia broncopulmonar conforme o peso de nascimento e os critérios diagnósticos (dependência de oxigênio com 28 dias de vida ou com 36 semanas de idade pós-conceptual) ${ }^{10}$

\begin{tabular}{lcc}
\hline Peso ao nascer & \multicolumn{2}{c}{ Dependência de oxigênio } \\
$\mathbf{2 8}$ dias & $\begin{array}{c}\mathbf{3 6} \text { semanas } \\
\text { pós-natal }\end{array}$ & pós-conceptual \\
\hline$<750 \mathrm{~g}$ & $90-100 \%$ & $54 \%$ \\
$750-999 \mathrm{~g}$ & $50-70 \%$ & $33 \%$ \\
$1.000-1.249 \mathrm{~g}$ & $30-60 \%$ & $20 \%$ \\
$1.250-1.499 \mathrm{~g}$ & $6-40 \%$ & $10 \%$ \\
\hline
\end{tabular}

\section{Fisiopatologia}

A patogênese da DBP é multifatorial (Figura 1). Acredita-se que esses diversos fatores atuem de forma aditiva ou sinérgica, gerando inflamação e lesão pulmonar ${ }^{8}$. A agressão ao tecido pulmonar em desenvolvimento resulta em fibrose e desorganização do processo maturativo normal.

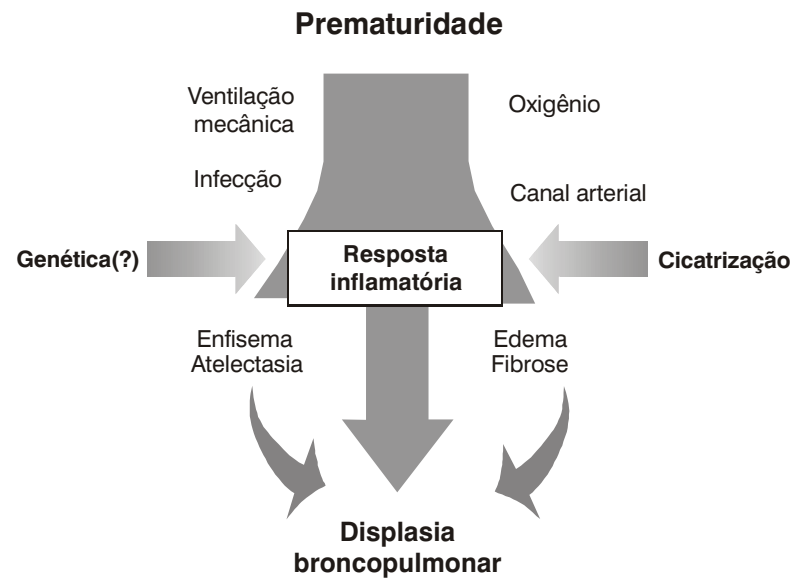

Figura 1 - Patogênese da displasia broncopulmonar ${ }^{1}$

\section{Fatores associados à lesão pulmonar}

Prematuridade: a prevalência da DBP é inversamente proporcional à idade gestacional de nascimento. A imaturidade pulmonar, portanto, é um dos fatores mais importantes na etiopatogenia da doença. A DBP representa a resposta de pulmões imaturos à lesão pulmonar aguda provocada pela ventilação mecânica, pelo oxigênio e por vários outros fatores. Sabe-se que os sistemas antioxidantes desenvolvem-se no último trimestre da gestação e, portanto, as enzimas antioxidantes têm menor atividade nos prematuros. Além disso, esses pacientes também apresentam níveis menores de antiproteases. Esses são alguns dos motivos pelos quais há uma menor capacidade de controle da 
inflamação nesses pacientes. Além disso, existem alterações na regulação dos mecanismos de reparação, favorecendo o aparecimento de fibrose nos segmentos acometidos. Não se sabe, porém, a forma exata pela qual a imaturidade pulmonar interfere no processo "inflamação $x$ reparação", produzindo lesões irreversíveis ${ }^{1,2,14}$.

Oxigênio: a lesão pulmonar induzida pelo oxigênio é deflagrada pela produção de radicais tóxicos, tais como superóxido, peróxido de hidrogênio e radicais livres. Como já relatado, o recém-nascido, especialmente o prematuro, é mais vulnerável a essas lesões, porque os sistemas antioxidantes (por exemplo a catalase e superóxido dismutase) ainda não se desenvolveram completamente. Os metabólitos ativos do oxigênio provocam grande dano tecidual através da oxidação de enzimas, inibição de proteases e da síntese de DNA, diminuição da síntese de surfactante e peroxidação de lipídeos, além de funcionarem como fatores quimiotáticos de células inflamatórias ${ }^{1,2,14}$.

Ventilação mecânica: os dois principais fatores relacionados com a lesão pulmonar induzida pela ventilação mecânica são a instabilidade alveolar gerando atelectasias (atelectrauma) e a hiperdistensão regional de alvéolos e vias aéreas (baro/volutrauma). O atelectrauma é a lesão provocada pelos ciclos repetidos de colapso e re-expansão alveolar durante a ventilação mecânica. O surgimento de áreas não recrutadas (atelectasias) não é apenas conseqüência, mas também causa de lesão pulmonar. Há evidências de que estratégias ventilatórias que utilizam baixas pressões ao final da expiração (PEEP) estão associadas a maior lesão pulmonar e menor eficácia da terapêutica com o surfactante exógeno. A ventilação, portanto, deve visar ao recrutamento alveolar, otimizando a capacidade residual funcional. Dessa forma, protege-se o pulmão contra o atelectrauma e reduz-se a necessidade de altas concentrações de oxigênio, minimizando-se o dano pulmonar. O volutrauma é a lesão associada a hiperdistensão das estruturas pulmonares provocada pelo uso de grandes volumes correntes durante a ventilação mecânica. Tal fato causa dano pulmonar por "estiramento" dos alvéolos, vias aéreas, membrana basal e até do endotélio capilar pulmonar. Há, então, aumento da permeabilidade capilar e extravasamento de fluidos, proteínas e sangue, gerando edema e inflamação. Além disso, a quebra da barreira alvéolo-capilar ocasionada pelo volutrauma pode permitir que mediadores inflamatórios e bactérias penetrem na circulação e provoquem reação inflamatória sistêmica e infecção em outros órgãos. O atelectrauma e o volutrauma contribuem de forma sinérgica para o dano pulmonar. Atualmente, sabese que PEEP insuficiente (predispondo ao atelectrauma) e o uso de grandes volumes correntes são as principais causas de lesão pulmonar aguda induzida pela ventilação mecânica. O barotrauma é a lesão pulmonar provocada pelo uso de altas pressões durante a ventilação mecânica. Pesquisas em modelos experimentais que utilizaram altas pressões ventilatórias sem grandes volumes correntes causaram relativamente pouca lesão pulmonar. Atualmente, acredita-se que a pressão, de forma isolada, não seja considerada um fator preponderante na gênese da lesão pulmonar $2,8,14,15$.
Infecção: existem evidências de que a infecção pré- e pós-natal possam contribuir para o desenvolvimento da DBP por causa da liberação de mediadores inflamatórios e afluxo de células inflamatórias no pulmão. Alguns estudos correlacionam a ocorrência de corioamnionite com o desenvolvimento da DBP. Outros sugerem a associação de DBP com a infecção pelo Ureaplasma urealyticum, mas não está comprovado se essa relação é do tipo causal. Além de provocar liberação de mediadores pró-inflamatórios, a infecção pulmonar, seja congênita ou nosocomial, pode gerar necessidade de suporte ventilatório, que aumenta ainda mais o dano pulmonar1,14-17.

Persistência do canal arterial (PCA): a PCA ocasiona um aumento do fluxo sangüíneo pulmonar e edema intersticial, gerando diminuição da complacência pulmonar e aumento da resistência de via aérea. Isso pode resultar numa estratégia ventilatória mais agressiva e prolongada, aumentando, assim, o risco de aparecimento da DBP1.

Fatores genéticos: acredita-se que exista uma predisposição genética para o desenvolvimento da DBP. O mecanismo ainda não está esclarecido, mas pode estar relacionado ao processo de reparação pulmonar, com maior ou menor estímulo para a reestruturação do tecido pulmonar normal ou para a proliferação de fibroblastos e fibrose. Sugere-se que os genes reguladores do desenvolvimento pulmonar sofram interferência dos mediadores inflamatórios presentes no pulmão lesado (por exemplo, o fator beta de transformação de crescimento - TGF- $\beta$ ), provavelmente estimulando a expressão gênica de mediadores que favorecem o surgimento de fibrose ${ }^{1,8}$.

Desnutrição e deficiência de vitamina A: o estado nutricional preservado e a vitamina A contribuem para o processo de diferenciação, regeneração e reepitelização do tecido pulmonar, sendo sugerida por alguns autores a associação desses componentes com a DBP1,18,19.

A inflamação é a via final comum dos fatores que provocam lesão pulmonar. O processo inflamatório ocorre pela agressão ao epitélio respiratório e ao endotélio capilar pulmonar, provocando liberação de mediadores pró-inflamatórios e citocinas quimiotáticas. Ocorre afluxo de células inflamatórias, principalmente de granulócitos, que potencializam a reação inflamatória com a produção de mais citocinas (interleucina-8, proteína inflamatória macrofágica-1, TGF-ß e outras) e liberação de enzimas (por exemplo, a elastase). Essas substâncias produzem aumento da permeabilidade vascular, que contribui para o edema intersticial, alveolar e da via aérea. É importante lembrar o fato de que a circulação pulmonar não só recebe todo o débito cardíaco, como também abriga um reservatório de neutrófilos. O pulmão, portanto, contribui para a circulação de células e mediadores inflamatórios para todo o organismo $1,14,15,20,21$.

\section{Cicatrização}

A resolução do processo inflamatório pode seguir dois caminhos: reparação das estruturas pulmonares com restauração da função pulmonar normal, ou fibrose, levando a 
prejuízos da função respiratória. A tendência de seguir por um ou outro caminho depende de vários fatores, como estado nutricional, mediadores inflamatórios, genéticos, entre outros, conforme já descrito.

No processo de reparação pulmonar, observa-se a reepitelização e reestruturação do parênquima pulmonar. Os pneumócitos tipo II têm o importante papel de diferenciação em pneumócitos tipo I, substituindo as células lesadas. Já no processo de fibrose, o macrófago alveolar é considerado como o principal responsável pela liberação das citocinas que levam à proliferação de fibroblastos e deposição de colágeno. Parece haver um espectro variável entre os dois processos no mesmo paciente, em diferentes regiões pulmonares, o que poderia justificar a grande variabilidade clínica da doença ${ }^{1,8,15}$.

\section{Desenvolvimento pulmonar e DBP}

Uma série de eventos perinatais tem sido associada à ocorrência da DBP, com especial atenção ao processo de desenvolvimento do sistema respiratório. O desenvolvimento pulmonar completa-se nos primeiros anos de vida, de tal modo que mesmo indivíduos nascidos a termo estão vivenciando intensamente o período de alveolarização do tecido pulmonar. Os nascimentos antes do termo podem ocorrer durante a fase canalicular do desenvolvimento pulmonar, que compreende o período da $17^{a}$ a $24^{a}$ semana de gestação, ou durante as fases de saculação terminal e alveolar (a partir da $25^{a}$ semana). Na fase canalicular, ocorre a maturação das vias condutoras pulmonares e inicia-se o desenvolvimento das unidades respiratórias terminais, que irão amadurecer nas fases sacular e alveolar, preparando o pulmão para a troca gasosa. A lesão durante a formação do parênquima pulmonar pode resultar em uma alteração na progressão do processo de alveolarização, resultando num quadro de simplificação alveolar. Dessa forma, a DBP representa a ruptura do processo normal de desenvolvimento e reparação dos pulmões em resposta à lesão aguda pulmonar ${ }^{1,8,14,15}$.

\section{Quadro clínico}

O quadro clínico consiste em sintomas respiratórios associados à dependência ao oxigênio e alterações radiológicas em recém-nascidos, em geral prematuros submetidos a ventilação mecânica. A ausculta pulmonar pode ser pobre, às vezes notando-se apenas taquidispnéia. Em casos mais graves, a hipoxemia pode estar acompanhada de hipercapnia. Muitos pacientes apresentam deformidade torácica, taquidispnéia de graus variados e menor tolerância aos exercícios físicos. Tosse e crises de sibilância são freqüentes. A sintomatologia é extremamente variável e depende da gravidade da $\mathrm{DBP}^{1,15}$.

\section{Quadro radiológico}

As alterações radiológicas podem variar de hiperinsuflação pulmonar com espessamento brônquico e atelectasias até a presença de traves opacas de fibrose, grandes cistos e enfisema intersticial. Pode ser evidente o tronco da artéria pulmonar pela hipertensão pulmonar associada e, em casos muito graves, o aumento da área cardíaca. Mais de $90 \%$ dos pacientes com DBP apresentam alterações radiológicas à tomografia computadorizada de alta resolução ${ }^{1,15,22}$.

\section{Aspectos anatomopatológicos}

$\mathrm{Na}$ "era pré-surfactante" os achados anatomopatológicos da DBP caracterizavam-se pelo predomínio de processo inflamatório crônico e fibrose do parênquima pulmonar, além da metaplasia epitelial escamosa e hipertrofia de músculo liso das vias aéreas. Como a lesão pulmonar e o quadro clínico eram mais graves, o acometimento do sistema cardiovascular era também mais proeminente, com proliferação da camada íntima, hipertrofia da camada muscular e do ventrículo direito (cor pulmonale). Essa "DBP clássica" é pouco comum nos dias atuais, devido ao uso do corticóide antenatal, da reposição neonatal de surfactante, de estratégias ventilatórias menos agressivas e à melhoria dos cuidados de suporte do recém-nascido prematuro. Ainda pode ser observada, entretanto, em pacientes que necessitam de suporte ventilatório agressivo e prolongado, como na síndrome de aspiração meconial grave, hipoplasia pulmonar associada à hérnia diafragmática e pneumonia congênita acompanhadas de hipertensão pulmonar, entre outras disfunções.

A partir da década de 90, com os avanços nos cuidados perinatais, observou-se maior sobrevivência de recémnascidos prematuros extremos. Os pulmões desses pacientes encontram-se na fase canalicular ou sacular de desenvolvimento, conforme já descrito. Ao nascimento, mesmo sofrendo poucas agressões, observa-se uma interrupção ou inibição do processo normal de desenvolvimento e crescimento pulmonar. Acredita-se que ocorra uma interferência nos processos sinalizadores do desenvolvimento pulmonar, prejudicando a maturação terminal e o curso da alveolarização, provavelmente por um defeito na formação dos septos secundários. Evidencia-se, então, uma simplificação nas áreas distais, com menor número de alvéolos. Notamse menos fibrose, mais tecido elástico e menor grau de lesão de vias aéreas. Além disso, ocorre inibição do desenvolvimento da microvasculatura pulmonar, o que contribui para o aumento da resistência vascular encontrada nesses pacientes. Esse quadro é conhecido, atualmente, como "nova DBP" (Tabela 3) 1,8,14,15.

\section{Função pulmonar}

As alterações funcionais pulmonares ocorrem também em graus variados e consistem em:

- aumento da resistência das vias aéreas e limitação do fluxo aéreo, levando à hiper-responsividade brônquica;

- aumento do trabalho respiratório;

- diminuição da complacência pulmonar pela fibrose, hiperinsuflação e atelectasias;

- aumento do volume residual e diminuição da capacidade residual funcional; 
Tabela 3 - Características da "nova displasia broncopulmonar" e da "displasia broncopulmonar clássica ou velha"

\begin{tabular}{ll}
\hline Tipo de displasia & Características \\
\hline "Nova displasia broncopulmonar" & Menor hipertrofia da musculatura lisa \\
& Menos fibrose \\
& Metaplasia escamosa menos grave \\
& Menor número e maior diâmetro alveolar (defeito da septação?) \\
& Microvasculatura pulmonar dismórfica \\
& Aumento do tecido elástico \\
"Displasia broncopulmonar clássica" & Metaplasia do epitélio respiratório \\
& Hipertrofia da musculatura lisa \\
& Fibrose significativa \\
& Grandes alterações vasculares \\
\hline
\end{tabular}

- hipoxemia, agravada por fatores como sono, agitação, alimentação e infecções;

- hipercapnia em casos mais graves.

A persistência dessas alterações funcionais é variável, conforme descrito adiante ${ }^{1,22-24}$.

\section{Prevenção da DBP}

A principal forma é a prevenção do parto prematuro, com acompanhamento pré-natal adequado2,8,14. Entre outras medidas preventivas, destacam-se as seguintes:

\section{Corticosteróide}

Antenatal: os efeitos benéficos do uso antenatal de corticosteróide já está bem estabelecido. A administração desse fármaco à gestante antes do parto prematuro diminui a incidência de SDR, hemorragia peri/intraventricular e mortalidade neonatal. O corticóide parece aumentar a quantidade de enzimas antioxidantes e modula a diferenciação de vários tecidos fetais, preparando o concepto para o nascimento prematuro. Os efeitos desse fármaco parecem ser amplificados quando, à corticoterapia materna, associase a terapêutica de reposição com o surfactante exógeno ao prematuro logo após o nascimento, diminuindo bastante a necessidade de suporte ventilatório agressivo. Apesar de não comprovado por estudos controlados, acredita-se que o uso do corticóide possa diminuir a incidência e a gravidade da DBP. Assim, o uso do corticosteróide deve ser considerado em todas gestantes que iniciem trabalho de parto prematuro entre 24 a 34 semanas de idade gestacional.

Pós-natal: observa-se na literatura que as indicações para o uso da corticoterapia sistêmica na fase pós-natal são altamente controversas e variam entre os diversos serviços ${ }^{25-27}$. Existem evidências de que o processo inflamatório tenha uma participação importante na patogênese da DBP. Tal fato tem propiciado o uso de terapias que possam reduzir ou modular o processo inflamatório pulmonar na tentativa de diminuir a incidência ou a gravidade da DBP. A corticoterapia reduz o processo inflamatório, o edema e a fibrose, além de induzir a broncodilatação, mecanismos esses responsáveis pela rápida melhora da função pulmonar após o seu uso, permitindo a retirada da ventilação mecânica. Por essa razão, o corticóide foi considerado uma alternativa na prevenção e no tratamento da DBP. Estudos demonstraram que o corticosteróide sistêmico reduz de fato a incidência de DBP e permite uma extubação mais precoce. Entretanto, alterações neurológicas tardias têm sido observadas na evolução desses pacientes, especialmente com o uso precoce da dexametasona em doses elevadas. Uma metanálise recente ${ }^{28}$ concluiu que o uso de corticosteróide em recémnascidos prematuros, apesar dos benefícios acima mencionados, não alterou a mortalidade e nem o tempo de permanência hospitalar. Os benefícios em curto prazo podem não superar os graves riscos em longo prazo da corticoterapia pós-natal, em especial no que se refere às alterações neurológicas. Dessa forma, sugere-se que a administração sistêmica do corticóide no período neonatal deve ser restrita a situações excepcionais e ter o consentimento informado dos pais 26,28-32. Apesar disso, há ainda controvérsia em relação ao uso pós-natal do corticosterói$\mathrm{de}^{33}$. Considera-se o seu uso para os neonatos prematuros dependentes de ventilação mecânica por mais de 7 dias com sinais radiológicos compatíveis com DBP e que preencham os seguintes critérios:

- necessidade de $\mathrm{FiO}_{2}$ maior que 0,40 e pressão média de vias aéreas acima de $10 \mathrm{cmH}_{2} \mathrm{O}$; e

- evidências clínicas e radiológicas de atelectasias de repetição, edema pulmonar recorrente sem resposta aos diuréticos ou presença de hiper-responsividade brônquica (sibilância, hipersecreção pulmonar).

É importante que sejam verificadas e corrigidas, antes de se iniciar a corticoterapia sistêmica, intercorrências clínicas que poderiam estar contribuindo para a gravidade do quadro respiratório, como: PCA com repercussão hemodinâmica, síndrome de escape de ar, processo infeccioso, impulsos respiratórios ineficientes (imaturidade do centro respiratório ou lesão de sistema nervoso central), distúrbios metabólicos e desnutrição. 
Recomendamos o uso da dexametasona por via intravenosa ou oral na seguinte posologia: $0,15 \mathrm{mg} / \mathrm{kg} / \mathrm{dia}$ a cada 12 horas por 3 dias; a seguir, 0,10 mg/kg/dia a cada 12 horas por 3 dias, e, finalmente, 0,05 mg/kg/dia a cada 12 horas por 3 dias. Se houver resposta positiva, com diminuição dos parâmetros ventilatórios (suporte de pressão e $\mathrm{FiO}_{2}$ ) após as doses iniciais (3 primeiros dias), continua-se o tratamento realizando o esquema completo. Caso não ocorra boa resposta durante os primeiros dias, deve-se suspender a terapêutica com o corticóide. Além disso, mesmo que os pacientes preencham os critérios de indicação da corticoterapia, devem ser evitados esquemas repetidos da droga, procurando-se alternativas para o controle do quadro respiratório.

Quanto ao uso da via inalatória para a administração do corticosteróide em neonatos, alguns autores relataram melhora na taxa de extubação e redução da necessidade de corticoterapia sistêmica. Entretanto, há poucos estudos sobre o uso do corticóide inalado como medicação preventiva da DBP, impossibilitando qualquer conclusão até o momento $30,34-39$.

\section{Surfactante}

A terapia com surfactante exógeno promove um recrutamento alveolar mais homogêneo, estabiliza as vias áreas terminais, reduzindo as atelectasias e favorecendo a diminuição dos parâmetros ventilatórios. Em recém-nascidos prematuros extremos, as evidências indicam vantagens do uso profilático (antes de 2 horas de vida) sobre o terapêutico na redução da mortalidade e da freqüência da DBP. Apesar do uso do surfactante exógeno não ter reduzido a incidência, acredita-se que tenha diminuído a gravidade da DBP2.

\section{Estratégias ventilatórias}

O objetivo da ventilação mecânica deve abranger a minimização da lesão pulmonar, através do recrutamento de áreas em colapso e otimização da capacidade residual funcional, sem promover hiperdistensão das áreas já recrutadas. Dessa forma, tenta-se minimizar o atelectrauma e o baro/volutrauma. Várias estratégias ventilatórias visando à redução da lesão pulmonar e, conseqüentemente, a DBP, têm sido descritas, destacando-se: ventilação convencional com estratégias de proteção pulmonar (baixos volumes correntes -4 a $6 \mathrm{ml} / \mathrm{kg}$, otimização da PEEP e hipercapnia permissiva), posição prona, pressão positiva contínua na via aérea (CPAP) nasal e ventilação de alta freqüência. Até o momento, entretanto, estudos controlados com o emprego dessas estratégias ainda não comprovaram de forma evidente a redução da incidência da DBP. Além disso, a hipercapnia permissiva precisa ser mais estudada nos prematuros, pelo possível risco de provocar lesão em sistema nervoso central2,8,14,40,41.

\section{Vitamina A}

A vitamina A atua na diferenciação e manutenção da integridade do epitélio respiratório. Sua deficiência leva à inibição do processo de alveolarização e à diminuição do transporte mucociliar com aumento da metaplasia escamosa. Com base nesses dados, algumas pesquisas têm mostrado uma redução na incidência de DBP nos recémnascidos de risco suplementados com altas doses de vitamina $A^{1,18,19}$.

\section{Óxido nítrico inalatório}

O óxido nítrico é um potente vasodilatador e vem sendo utilizado por via inalatória durante a ventilação mecânica com o objetivo de diminuir o shunt pulmonar e a inflamação. Vários estudos vêm sendo conduzidos utilizando precocemente o óxido nítrico em baixas doses em recém-nascidos prematuros com hipertensão pulmonar, visando à prevenção da DBP. Até o momento, os resultados são inconclusivos ${ }^{8}$.

\section{Superóxido dismutase}

Com base nas evidências de que os prematuros são deficientes de sistemas antioxidantes endógenos e que os radicais livres do oxigênio desempenham importante papel na etiopatogenia da DBP, vários estudos têm utilizado a terapia antioxidante, em especial, a superóxido dismutase na prevenção da DBP. Estudos em modelos experimentais relatam efeitos benéficos do uso traqueal da superóxido dismutase recombinante humana, diminuindo o risco de lesão induzida pela hiperoxia. Dessa forma, é possível que o uso desse fármaco possa exercer algum papel na prevenção da DBP. São necessários mais estudos, pois ainda existem controvérsias $2,8,42,43$.

\section{Restrição hídrica}

Alguns estudos demonstraram que uma leve restrição hídrica nos prematuros extremos pode reduzir a freqüência de DBP, pois o edema pulmonar é um fator importante na fisiopatologia da doença².

\section{Tratamento}

O acompanhamento clínico de pacientes com DBP deve ser preferencialmente feito por uma equipe multidisciplinar, incluindo o neonatologista e outras subespecialidades pediátricas, como pneumologia, cardiologia, oftalmologia e neurologia, além de fisioterapeuta, nutricionista, fonoaudiólogo e eventualmente outros profissionais. O tratamento também deve ser individualizado, devido às variações na apresentação clínica e na gravidade. Os objetivos do tratamento estão apresentados na Tabela 41,2,8,10,44.

Oxigenoterapia: um dos pontos mais importantes no tratamento dos pacientes com DBP é a manutenção de níveis adequados de oxigênio arterial. A hipoxemia é a principal causa das alterações cardiovasculares (hipertensão pulmonar e cor pulmonale), além de influenciar o ganho ponderal e o desenvolvimento cerebral nos pacientes com DBP. Quando não corrigida, a hipoxemia também se correlaciona com um maior risco para ocorrência de morte súbita e episódios de apnéia nesses lactentes ${ }^{10,24}$. 
A suplementação de oxigênio deve ser realizada preferencialmente através de cânula nasal, visando manter os níveis de saturação de oxi-hemoglobina entre 92 e 95\%. Nos pacientes portadores de cor pulmonale, são recomendados valores maiores, entre 95 e 96\%. Apesar de haver certa controvérsia a respeito do melhor nível de oximetria desejável para os pacientes com DBP, sabe-se que esses valores devem ser mantidos estáveis durante a alimentação, o sono ou a vigília, evitando-se, portanto, a suplementação intermitente ${ }^{10,24}$.

Tabela 4 - Objetivos do tratamento da displasia broncopulmonar

- Promover o controle da sintomatologia

- Manter níveis de atividade normais

- Garantir crescimento somático e desenvolvimento neuropsicomotor adequados

- Manter a função pulmonar tão normal quanto possível

- Minimizar as exacerbações da doença

- Prevenir e intervir precocemente nas infecções respiratórias

- Evitar os efeitos colaterais das medicações

- Tentar manter o paciente em casa
Nievas \& Chernick ${ }^{10}$, em 2002, sugeriram um algoritmo para o uso de oxigênio na DBP, demonstrado na Figura 2. Inicialmente, avalia-se a necessidade de oxigênio com o paciente em estado de alerta. O oxímetro de pulso é utilizado para medir a saturação em paciente acordado a cada 2 a 3 semanas, após 10 minutos respirando ar ambiente, titulando-se a necessidade de cada indivíduo. Se a saturação mantiver-se igual ou maior que $92 \%$ (ou $95 \%$, se cor pulmonale), a suplementação de oxigênio poderá ser suspensa durante os períodos de vigília, mantendo-se ainda durante o sono. A seguir, passa-se a medir a saturação durante o sono, preferencialmente com aparelhos capazes de registrar a oximetria durante a noite ${ }^{23}$. Recomenda-se suspender a oxigenoterapia quando os níveis descritos acima forem atingidos. Caso haja baixo ganho ponderal em qualquer etapa descrita acima, na presença de boa aceitação de dieta hipercalórica, a suplementação de oxigênio por período integral deverá ser reiniciada, pois isso é um forte indício de presença de episódios intermitentes de hipoxemia significante. Se a dependência de oxigênio persistir por vários meses após a alta da UTI neonatal, devem ser procuradas condições coexistentes que poderiam estar piorando o quadro da DBP ou mimetizando-a, como: refluxo gastroesofágico ainda não tratado, episódios de microaspiração secundários à incoordenação cricofaríngea, cardiopatia congênita não diagnosticada, entre outras ${ }^{1,2,10,24}$.

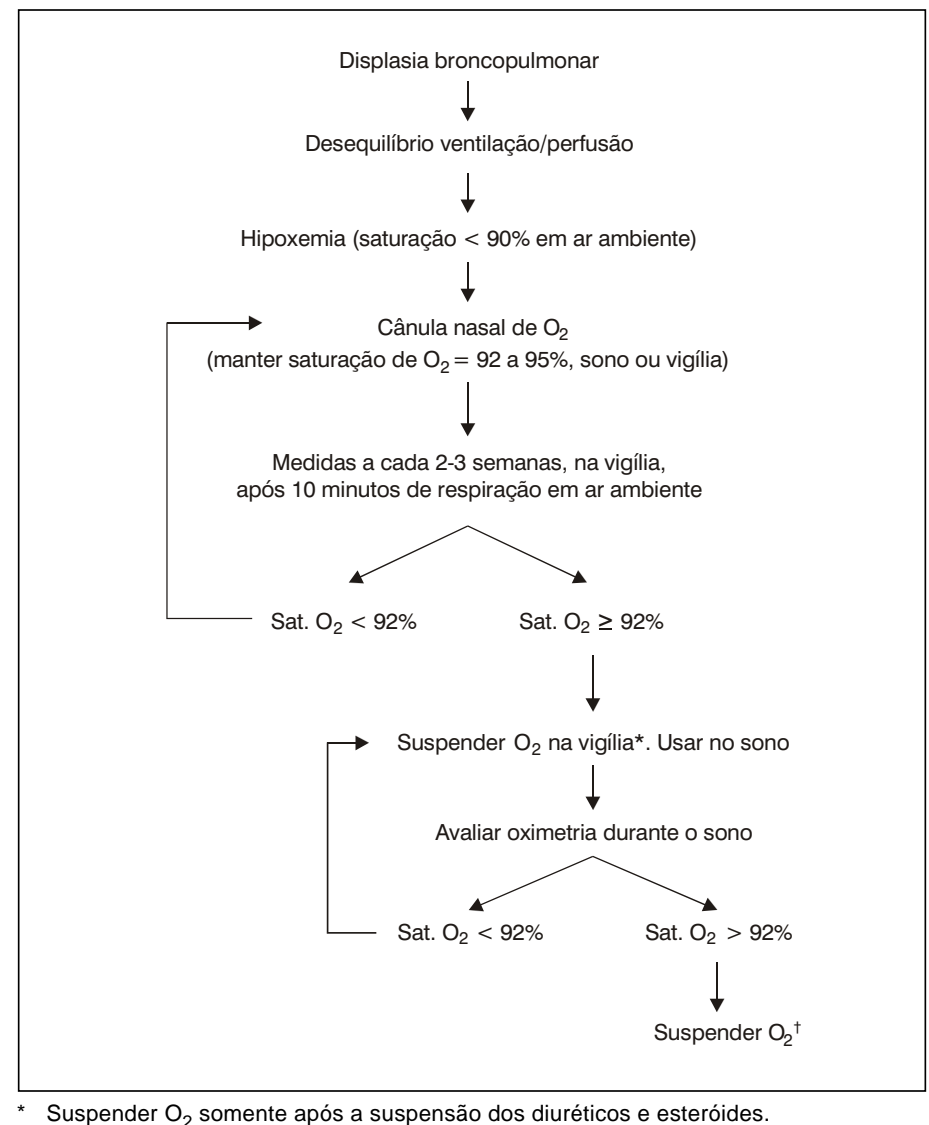

* Suspender $\mathrm{O}_{2}$ somente após a suspensão dos diuréticos e esteróides.

Figura 2 - Algoritmo de oxigenioterapia em displasia broncopulmonar ${ }^{10}$ 
Recomendamos manter a suplementação de oxigênio por cânula nasal até que seja possível a suspensão de medicamentos como diuréticos, e o paciente apresente-se estável clinicamente e com saturação de oxigênio maior ou igual a $93 \%$ durante todo o dia. A suspensão da oxigenoterapia noturna ocorre posteriormente à retirada da suplementação diurna na maioria das vezes.

Diuréticos: alguns pacientes cursam com edema pulmonar recorrente ou função cardíaca comprometida. Nessas situações, indica-se a terapia com diuréticos, preferencialmente a furosemida, pois alguns autores acreditam que, além do efeito diurético, essa droga tenha um efeito pulmonar direto, melhorando a complacência e a resistência de vias aéreas. No entanto, deve-se ter o cuidado para a identificação e correção dos efeitos colaterais da medicação, como os distúrbios hidroeletrolíticos, hipercalciúria, nefrocalcinose, entre outros. A terapia de curta duração mostra-se eficaz no controle do edema pulmonar; entretanto, a eficácia do uso prolongado de diuréticos é controver$\mathrm{sa}^{1,2,10,24,45,46}$. Assim, considera-se o uso da furosemida nos casos de edema pulmonar recorrente e sintomático e para possibilitar maior oferta hídrica com o objetivo de aumentar a oferta calórica. Sugere-se iniciar com 1 a $2 \mathrm{mg} / \mathrm{kg} /$ dose, duas vezes ao dia, por via intravenosa ou oral. A retirada do diurético é indicada quando o paciente demonstra melhora do quadro pulmonar. Nos casos em que haja necessidade de manutenção do diurético por mais tempo, recomendamos o uso da associação de hidroclorotiazida e espironolactona na dose de 2 a $4 \mathrm{mg} / \mathrm{kg}$ por dia ou o uso de furosemida em dias alternados, visando minimizar os efeitos adversos. $O$ uso de diuréticos não traz benefício evidente em pacientes com idade superior a 6 meses, exceto quando existirem indicações adicionais (nefrocalcinose, cor pulmonale).

Corticosteróides: uma vez instalada a DBP, os corticóides são indicados por via inalatória (inalador dosimetrado pressurizado com espaçador) no controle da hiperresponsividade brônquica $1,2,10$. Recomendamos o uso de corticosteróide inalado para os pacientes com dinâmica respiratória mais comprometida e padrão clínico-radiológico mais evidente de obstrução de vias aéreas inferiores. Nessa situação, optamos pelo uso de nebulímetros acoplados a espaçadores valvulados com máscara. Procuramos utilizar baixas doses de corticosteróide por períodos relativamente prolongados (mínimo de 6 meses de uso contínuo, aproximadamente).

Broncodilatadores: são indicados por via inalatória (inalador dosimetrado com espaçador) para o controle dos sintomas respiratórios (tosse, sibilância) quando há evidência clínica e/ou funcional de boa resposta. Sabe-se que, na DBP, há hipertrofia da musculatura lisa das vias aéreas; portanto, grande parte dos pacientes pode beneficiar-se do uso dessas medicações 1,2,10,24,47. O uso dos broncodilatadores orais deve ser evitado, pelo maior risco de efeitos colaterais.

Nutrição: vários fatores contribuem para a desnutrição dos pacientes portadores de DBP. Destacam-se o alto gasto energético, pelo aumento do trabalho respiratório, as alterações no metabolismo de glicose e lipídeos, pela hipoxemia crônica, e a diminuição da ingesta alimentar. Recomendase o uso de dieta hipercalórica (cerca de 110 a $150 \mathrm{kcal} / \mathrm{kg}$ por dia) com suplementos ricos em triglicérides de cadeia média e certa restrição de carboidratos naqueles pacientes retentores de gás carbônico. Em nosso meio, pode ser utilizada a suplementação com preparados de óleo de canola ou de girassol, que têm menor custo. Vale ressaltar que outras causas de deficiência de ganho ponderal podem estar presentes e devem ser investigadas e tratadas precocemente, como: hipoxemia, anemia, refluxo gastroesofágico, cardiopatia congênita e incoordenação à deglutição, entre outras $1,2,10,24,42,48$.

Higiene antiinfecciosa: os pacientes com DBP são vítimas de infecções recorrentes do trato respiratório. Além da vacinação habitual, recomenda-se, portanto, vacina antipneumocócica e vacinação anual antiinfluenza. Existe também maior risco de infecção pelo vírus respiratório sincicial (VRS) nos prematuros em comparação com as crianças de termo. Vários estudos demonstraram que a profilaxia com a imunoglobulina contra o VRS diminui o risco de internação e a gravidade das infecções por esse agente. Atualmente, a Academia Americana de Pediatria ${ }^{49}$ recomenda o uso da imunoglobulina intravenosa anti-VRS ou, preferencialmente, do anticorpo monoclonal intramuscular anti-VRS (palivizumab) durante as estações outono e inverno (quando o VRS é mais freqüente) nos pacientes portadores de DBP com idade inferior a 2 anos nas seguintes situações:

- pacientes que estão em tratamento com oxigênio; e

- pacientes que estiveram sob tratamento durante os 6 meses anteriores.

Tais pacientes devem receber o palivizumab durante os meses de outono e inverno em cinco doses de $15 \mathrm{mg} / \mathrm{kg}$ por dose, por via intramuscular, uma vez por mês. Vale salientar a contra-indicação relativa do uso do palivizumab em lactentes com cardiopatias congênitas cianogênicas $1,2,10,50$. Nos dias atuais, não existe disponibilidade para a administração de profilaxia para VRS nas instituições públicas, pois ainda não há cobertura pelo Ministério da Saúde. É importante ressaltar que, apesar de já demonstrado nos EUA, até o momento não existem estudos brasileiros sobre a redução dos custos relacionados à saúde quando se utiliza esse tipo de prevenção.

Controle da hipertensão pulmonar e do cor pulmonale: a principal medida é o uso do oxigênio para o alívio da hipertensão pulmonar, já que as alterações cardiovasculares são secundárias às alterações pulmonares. Como terapêutica adicional, podem ser utilizados os diuréticos, bloqueadores do canal de cálcio e óxido nítrico, entre outros ${ }^{1}$.

\section{Critérios de alta hospitalar}

Sugerem-se os seguintes critérios para alta hospitalar dos recém-nascidos portadores de DBP: 
- saturação de oxi-hemoglobina ao redor de 95\% num fluxo de oxigênio estável ou em diminuição pelo menos nas últimas 2 semanas;

- ganho de peso satisfatório;

- ausência de episódios de apnéia pelo menos nas últimas 2 semanas;

- nenhuma necessidade de modificação nas medicações na última semana;

- possibilidade de retorno em visita ambulatorial em 48 a 72 horas;

- pais seguros em relação aos cuidados do lactente, inclusive no uso do oxigênio e/ou manipulação da sonda gástrica, em casos específicos, além da capacitação para o reconhecimento de sinais de piora e de como procederem em caso de emergência.

A realização de visita domiciliar da equipe antes da alta do lactente é importante para que se possa verificar e testar o equipamento necessário e avaliar o conhecimento das pessoas que irão cuidar da criança. É importante ainda avaliar as condições socioeconômicas da família, condições de moradia, acesso a telefone, transporte e proximidade a hospitais de referência1,3.

\section{Prognóstico e evolução}

O prognóstico desses pacientes é variável, dependendo da gravidade da doença. A morbimortalidade é maior no primeiro ano de vida, diminuindo nos anos seguintes $1,10,22-24$.

Pulmonar: os pacientes com DBP têm maior morbidade respiratória durante a vida, especialmente nos primeiros 2 anos de vida. Essa característica pode estender-se até mesmo à idade adulta. Aproximadamente $50 \%$ dos recém-nascidos de extremo baixo peso apresentam episódios recorrentes de sibilância e 33\% ainda persistem com sintomas na fase pré-escolar. Há relatos de que o risco de sibilância grave é minimizado em $10 \%$ para cada semana gestacional adicionada. O risco de recorrência de sintomas respiratórios aumenta se o paciente tiver irmãos, história familiar de atopia ou exposição ao fumo, sendo este, portanto, fortemente desencorajado entre os familiares. Além do maior risco para o desenvolvimento de sintomas respiratórios, os prematuros de extremo baixo peso com DBP apresentam uma taxa de readmissão hospitalar de $50 \%, 20$ vezes maior do que os pacientes nascidos a termo. A principal causa de internação é a infecção do trato respiratório inferior, especialmente de etiologia viral, daí a importância da higiene antiinfecciosa, mencionada acima. Em relação às alterações da função pulmonar descritas anteriormente, os pacientes apresentam doença obstrutiva de via aérea com aumento da sua resistência e aprisionamento aéreo, principalmente nos primeiros 2 anos de vida. Essas alterações são evidenciadas mesmo nos pacientes assintomáticos, porém em um menor grau. Pode-se ainda observar a ocorrência de deformidades torácicas, de maior ou menor intensidade. A capacidade residual funcional tende a normalizar-se entre 12 e 24 meses de idade. A complacência pulmonar, que entre 2 e 4 meses de idade é de apenas $35-50 \%$ do normal, aos 10 meses sobe para $60 \%$, normalizando-se por volta dos 3 anos de idade. 0 volume residual tem uma melhora lenta e pode normalizar-se somente entre os 8 e 10 anos, enquanto que a resistência de vias aéreas pode permanecer aumentada até a idade adulta. Estima-se que cerca de metade dessas crianças tenham sintomas respiratórios induzidos pelo exercício. Acredita-se que o crescimento pulmonar, propiciando a formação de novas unidades de troca gasosa, possa ocorrer até os 8 anos de idade. A dependência ao oxigênio, portanto, reverte-se na maioria dos pacientes. 0 impacto funcional dessas anormalidades aparentemente não é grande $1,10,22-24,51$.

Cardiovascular: evidências eletrocardiográficas de hipertrofia ventricular direita foram relatadas em $50 \%$ das crianças com DBP em idade escolar. Já em adolescentes e adultos jovens, raramente evidencia-se alguma alteração ${ }^{22}$.

Crescimento: os lactentes, em geral, com cerca de apenas 18 meses já atingem a curva de crescimento em estatura, porém o chamado catch-up pode ocorrer até pelo menos os 10 anos de idade. Em estudos de acompanhamento em curto prazo, há descrições de deficiência em peso e altura de crianças com DBP quando comparadas ao grupo sem a doença. Estudos evolutivos em longo prazo não confirmaram essa diferença em crianças entre 8 e 10 anos de idade. Atualmente, considera-se que o atraso no crescimento relaciona-se mais com a prematuridade e baixo peso ao nascer do que com o fato de esses pacientes serem portadores de $\mathrm{DBP}^{22-24}$.

Desenvolvimento: a freqüência de alterações neurológicas em crianças que tiveram DBP varia de 0 a $60 \%$ aos 24 meses de idade. Essa alta taxa de variabilidade reflete as divergências quanto à definição utilizada, variações socioeconômicas e diferentes práticas na assistência perinatal, além do espectro de gravidade da doença. Sabe-se que os pacientes com doença mais grave, especialmente os que precisaram de longo tempo de ventilação mecânica e de corticosteróide sistêmico, evoluem de forma menos satisfatória. Apesar da presença das alterações cognitivas, sensoriais, motoras e de linguagem enquanto lactentes, as crianças geralmente melhoram muito com a idade. Dessa forma, é importante o acompanhamento neurológico dos pacientes com $\mathrm{DBP}^{23,52}$.

Deve-se lembrar que a maioria dos estudos de seguimento em longo prazo refere-se a pacientes portadores da DBP da era pré-surfactante, portanto mais graves. Até o momento, existem poucos dados sobre o prognóstico tardio dos pacientes pertencentes ao grupo da "nova DBP".

\section{Conclusão}

A DBP vem sendo profundamente estudada na tentativa de identificação das suas causas e das possibilidades de prevenção e de tratamento. Ainda existem controvérsias a 
esse respeito e também em relação ao prognóstico desses pacientes, especialmente quando se trata da evolução tardia da "nova" DBP. É prioridade futura a identificação de estratégias para prevenção e tratamento do processo desencadeante da DBP. Existe, portanto, um amplo campo para pesquisa nesse assunto.

Finalmente, deve-se destacar a importância do acompanhamento dos pacientes com DBP por equipe multidisciplinar. Em nosso meio, existe grande dificuldade no tratamento global dos pacientes pertencentes a classes socioeconômicas menos favorecidas, pelas precárias condições de moradia, maior número de pessoas na casa, dificuldade de meios de transporte às unidades de saúde e hospitais, insuficiência de recursos para a compra de alimentos e medicações, entre outros fatores. Daí a importância de profissionais de várias áreas trabalhando em conjunto, com o objetivo de permitir crescimento e desenvolvimento adequados, com ênfase em proporcionar melhor qualidade de vida para esses pacientes e seus familiares.

\section{Agradecimentos}

Agradecemos à Suely Dornellas do Nascimento, médica assistente da disciplina de Pediatria Neonatal da Universidade Federal de São Paulo (UNIFESP/EPM), pelo seu auxílio na revisão do artigo na sua fase final de elaboração.

\section{Referências}

1. Silva Filho LVF. Doença pulmonar crônica neonatal. J Pediatr (Rio J). 1998;74:265-74.

2. Barrington $\mathrm{KJ}$, Finer NN. Treatment of bronchopulmonary dysplasia. A review. Clin Perinatol. 1998;25:177-202.

3. Hazinski TA. Bronchopulmonary dysplasia. In: Chernik, V, editor. Disorders of the respiratory tract in children. Philadelphia: WB Saunders; 1990. p. 300-20.

4. Northway Jr WH, Rosan RG, Porter DY. Pulmonary disease following respiratory therapy of hyaline-membrane disease. Bronchopulmonary dysplasia. N Engl J Med. 1967;276:357-68.

5. Pursey VA, Macpherson RI, Chernick V. Pulmonary fibroplasia following prolonged artificial ventilation of the newborn infant. Can Med Assoc J. 1969;100:451-7.

6. Bancalari E, Abdenour GE, Feller R, Gannon J. Bronchopulmonary dysplasia: clinical presentation. J Pediatr. 1979;95:819-23.

7. Shennan AT, Dunn MS, Ohlsson A, Lennox K, Hoskins EM. Abnormal pulmonary outcomes in premature infants: prediction from oxygen requirement in the neonatal period. Pediatrics. 1988;82:527-32.

8. Jobe AH, Bancalari E. Bronchopulmonary dysplasia. Am J Respir Crit Care Med. 2001;163:1723-9.

9. Lee SK, McMillan DD, Ohlsson A, Pendray M, Synnes A, Whyte $R$, et al. Variations in practice and outcomes in the Canadian NICU network 1996-1997. Pediatrics. 2000;106:1070-9.

10. Nievas FF, Chernick V. Bronchopulmonary dysplasia: an update for the pediatrician. Clin Pediatr. 2002;41:77-85.

11. Rojas MA, Gonzales A, Bancalari E, Claure N, Poole C, Silva-Neto G. Changing trends in the epidemiology and pathogenesis of neonatal chronic lung disease. J Pediatr. 1995;126:605-10.

12. Sinkin RA, Cox C, Phelps DL. Predicting risk for bronchopulmonary dysplasia: selection criteria for clinical trials. Pediatrics. 1990;86:728-36.

13. Cunha GS, Mezzacappa FF, Ribeiro JD. Fatores maternos e neonatais na incidência de displasia broncopulmonar em recémnascidos de muito baixo peso. J Pediatr (Rio J). 2003;79:550-6.
14. Clark RH, Gerstmann DR, Jobe AH, Moffitt ST, Slutsky AS, Yoder BA. Lung injury in neonates: causes, strategies for prevention, and long-term consequences. J Pediatr. 2001;139:478-86.

15. Jobe $A H$, Ikegami M. Mechanisms initiating lung injury in the preterm. Early Hum Dev. 1998;53:81-94.

16. van Waarde WM, Brus F, Okken A, Kimpen JL. Ureaplasma urealyticum colonization, prematurity and bronchopulmonary dysplasia. Eur Respir J. 1997;10:886-90.

17. Lyon A. Chronic lung disease of prematurity. The role of intrauterine infection. Eur J Pediatr. 2000;159:789-802.

18. Darlow BA, Graham PJ. Vitamin A supplementation for preventing morbidity and mortality in very low birthweight infants. Cochrane Database Syst Rev. 2002;(4):CD000501.

19. Verma RP, McCulloch KM, Worrell L, Vidyasagar D. Vitamin A deficiency and severe bronchopulmonary dysplasia in very low birthweight infants. Am J Perinatol. 1996;13:389-93.

20. De Dooy JJ, Mahieu LM, van Bever HP. The role of inflammation in the development of chronic lung disease in neonates. Eur J Pediatr. 2001;160:457-63.

21. Papoff P. Infection, neutrophils, and hematopoietic growth factors in the pathogenesis of neonatal chronic lung disease. Clin Perinatol. 2000;27:717-31.

22. Eber E, Zach MS. Paediatric origins of adult lung disease. Thorax. 2001;56:317-23.

23. Greenough A. Measuring respiratory outcome. Semin Neonatol. 2000;5:119-26.

24. Primhak RA. Discharge and aftercare in chronic lung disease of the newborn. Semin Neonatol. 2003;8:117-25.

25. Truffert P, Empana JP, Breart G, Saugstad OD, Goelz R, Halliday $\mathrm{HL}$, et al. Treatment strategies for bronchopulmonary dysplasia with postnatal corticosteroids in Europe: the EURAIL survey. Acta Paediatr. 2003;92:948-51.

26. Halliday HL. Clinical trials of postnatal corticosteroids: inhaled and systemic. Biol Neonate. 1999;76(Suppl 1):S29-40.

27. Williams O, Greenough A. Post-natal corticosteroid use. Eur J Pediatr. 2003;162:613-15.

28. American Academy of Pediatrics - Committee on Fetus and Newborn - Canadian Paediatric Society - Fetus and Newborn Committee. Postnatal corticosteroids to treat or prevent chronic lung disease in preterm infants. Pediatrics. 2002;109:330-8.

29. Doyle L, Davis P. Postnatal corticosteroids in preterm infants: systematic review of effects on mortality and motor function. J Paediatr Child Health. 2000;36:101-7.

30. Halliday HL, Ehrenkranz RA, Doyle LW. Early postnatal $(<96$ hours) corticosteroids for preventing chronic lung disease in preterm infants. Cochrane Database Syst Rev. 2003;(1):CD001146.

31. Grier DG, Halliday HL. Corticosteroids in the prevention and management of bronchopulmonary dysplasia. Semin Neonatol. 2003;8:83-91.

32. Cole $\mathrm{CH}$. Postnatal glucocorticosteroid therapy for treatment and prevention of neonatal chronic lung disease. Expert Opin Investig Drugs. 2000;9:53-67.

33. Jobe $\mathrm{AH}$. Postnatal corticosteroids for preterm infants-do what we say, not what we do. N Engl J Med. 2004;350:1349-51.

34. Cole $\mathrm{CH}$, Colton $\mathrm{T}$, Shah BL, Abbasi S, MacKinnon BL, Demissie $\mathrm{S}$, et al. Early inhaled glucocorticoid therapy to prevent bronchopulmonary dysplasia. N Engl J Med. 1999;340:1005-10.

35. Shah V, OhIsson A, Halliday HL, Dunn MS. Early administration of inhaled corticosteroids for preventing chronic lung disease in ventilated very low birth weight preterm neonates. Cochrane Database Syst Rev. 2000;(2):CD001969.

36. Shah SS, Ohlsson A, Halliday $H$, Shah VS. Inhaled versus systemic corticosteroids for the treatment of chronic lung disease in ventilated very low birth weight preterm infants. Cochrane Database Syst Rev. 2003;(2):CD002057.

37. Barrington $\mathrm{KJ}$. The adverse neuro-developmental effects of postnatal steroids in the preterm infant: a systematic review of RCTs. BMC Pediatrics. 2001;1:1-14.

38. Cole $\mathrm{CH}$. Postnatal glucocorticoid therapy for prevention of bronchopulmonary dysplasia: routes of administration compared. Semin Neonatol. 2001;6:343-50.

39. Cole $\mathrm{CH}$. Inhaled glucocorticoid therapy in infants at risk for neonatal chronic lung disease. J Asthma. 2000;37:533-43.

40. Auten RL, Vozzelli M, Clark RH. Volutrauma. What is it, and how do we avoid it? Clin Perinatol. 2001;28:505-15.

41. Jobe AH, Ikegami M. Prevention of bronchopulmonary dysplasia. Curr Opin Pediatr. 2001;13:124-9. 
42. Shah PS. Current perspectives on the prevention and management of chronic lung disease in preterm infants. Paediatr Drugs. 2003;5:463-80.

43. Suresh GK, Davis JM, Soll RF. Superoxide dismutase for preventing chronic lung disease in mechanically ventilated preterm infants. Cochrane Database Syst Rev. 2001;(1): CD001968.

44. Ho LY. Bronchopulmonary dysplasia and chronic lung disease of infancy: strategies for prevention and management. Ann Acad Med Singapore. 2002;31:119-30.

45. Brion LP, Primhak RA. Intravenous or enteral loop diuretics for preterm infants with (or developing) chronic lung disease. Cochrane Database Syst Rev. 2002;(1):CD001453.

46. Brion LP, Primhak RA, Yong W. Aerosolized diuretics for preterm infants with (or developing) chronic lung disease. Cochrane Database Syst Rev. 2000;(2):CD001694. Review. Update in: Cochrane Database Syst Rev. 2001;(2):CD001694.

47. $\mathrm{Ng} \mathrm{GY,} \mathrm{Ohlsson} \mathrm{A.} \mathrm{Bronchodilators} \mathrm{for} \mathrm{the} \mathrm{prevention} \mathrm{and}$ treatment of chronic lung disease in preterm infants. Cochrane Database Syst Rev. 2001;(3):CD003214.

48. Atkinson SA. Special nutritional needs of infants for prevention and recovery from bronchopulmonary dysplasia. J Nutr. 2001; 131:S942-6.
49. American Academy of Pediatrics. Prevention of respiratory syncytial virus infections: indications for the use of palivizumab and update on the use of RSV-IGIV. Pediatrics. 1998;102:1211-6.

50. The Impact-RSV Study Group. Palivizumab, a humanized respiratory syncytial virus monoclonal antibody, reduces hospitalization from respiratory syncytial virus infection in highrisk infants. Pediatrics. 1998;102:531-6.

51. Kennedy JD. Lung function outcome in children of premature birth. J Paediatr Child Health. 1999;35:526-1.

52. Böhm B, Katz-Salamon M. Cognitive development at 5.5 years of children with chronic lung disease of prematurity. Arch Dis Child Fetal Neonatal Ed. 2003;88:F101-5.

Correspondência:

Luciana de Freitas Velloso Monte

SQSW 306, Bloco D - apto. 404, Sudoeste

CEP 70673-434 - Brasília, DF

E-mail: lucifv@icr.hcnet.usp.brou I-monte@ig.com.br 